\title{
Parametria da associação do midazolam ou diazepam em cães pré-tratados pela atropina e tratados pela dexmedetomidina e quetamina
}

\author{
Parametria of midazolam or diazepam combination in dogs pretreated with atropine and treated \\ with dexmedetomidine and ketamine
}

Eduardo Hatschbach ${ }^{1}$ Flávio Massone ${ }^{2}$ Gustavo José von Glehn Santos ${ }^{3}$ Suzane Lilian Beier ${ }^{3}$

\section{RESUMO}

Em Anestesiologia Veterinária, busca-se continuamente a contenção farmacológica para estabelecer tratamentos, cruentos ou não, permitindo manipulações seguras e duradouras, dispensando a anestesia geral sem alterar, sobremaneira, os parâmetros fisiológicos. Visou-se empregar a dexmedetomidina em associação com a quetamina e o midazolam ou diazepam. A técnica anestésica foi aplicada em 30 cães hígidos, machos ou fêmeas, com pesos de 10 a $15 \mathrm{~kg}$ e idades de 2 a 4 anos, distribuidos aleatoriamente em três grupos (GI, GII, GIII). O grupo I recebeu um prétratamento de atropina $\left(0,044 \mathrm{mg}^{\mathrm{kg}^{-1}} \mathrm{SC}\right)$, e após 15 minutos, a dexmedetomidina $\left(3 \mu \mathrm{g} \mathrm{kg}^{-1} \mathrm{IV}\right)$, em dose única, aplicada durante dois minutos e uma dose de manutenção de

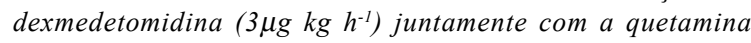
$\left(2 \mathrm{mg} \mathrm{kgh}^{-1}\right)$, completando-se para $20 \mathrm{ml}$ de solução fisiológica para infusão contínua, perfazendo uma hora de aplicação. $O$ grupo II recebeu o mesmo tratamento de GI, entretanto, recebeu juntamente com a dexmedetomidina, o midazolam $\left(0,2 \mathrm{mg} \mathrm{kg}^{-1}\right.$ IV) durante os dois minutos e outra dose, de $0,2 \mathrm{mg} \mathrm{kg} \mathrm{h} \mathrm{h}^{-1}$ junto com a dose de manutenção da dexmedetomidina e quetamina. O grupo III recebeu o mesmo tratamento de GII só que ao invés do midazolam, foi usado o diazepam $\left(0,5 \mathrm{mg} \mathrm{kg}^{-1} \mathrm{IV}\right)$ como dose de indução e $0,5 \mathrm{mg} \mathrm{kg} \mathrm{h} \mathrm{h}^{-1}$ junto com a dose de manutenção. Foi avaliado o efeito das benzodiazepinas frente à associação anestésica, além dos parâmetros respiratórios, oxicapnométricos, índice bispectral e periodo de recuperação. Após o término da colheita, a avaliação estatística foi efetuada por meio de Análise de Perfil. A partir dos resultados obtidos, pode-se concluir que a associação dos benzodiazepínicos promoveu sinergismo na sedação e miorrelaxamento, além de estabilidade cardiorrespiratória após pré-tratamento com atropina, sendo de grande valia como contenção farmacológica.
Palavras-chave: dexmedetomidina, quetamina, benzodiazepínicos, infusão contínua, cão.

\section{ABSTRACT}

Veterinary Anesthesiology is continuously in search of pharmacology containment to establish treatments, allowing safe and lasting manipulation, dispensing general anesthesia. The aim of the present study was to determine the use of dexmedetomidine in combination with ketamine and midazolam, or diazepam. The anesthetic technique was applied in 30 healthy dogs, male and female, weight between $10-15 \mathrm{~kg}$, and age between 2-4 years, divided in three groups (GI, GII and GIII). Group I (GI) received a pretreatment with atropine $\left(0.044 \mathrm{mg} \mathrm{kg}^{-1}, \mathrm{SC}\right)$, and 15 minutes later, a single dose of dexmedetomidine at $3 \mu \mathrm{g} \mathrm{kg}$, IV (induction), during two minutes. For maintenance was induced the same dose of dexmedetomidine $\left(3 \mu \mathrm{g} \mathrm{kg} \mathrm{h}^{-1}\right)$ combined with the ketamine $\left(2 \mathrm{mg} \mathrm{kg} \mathrm{h}^{-1}\right)$, all dilute in $20 \mathrm{ml}$ of distilled water, during onehour infusion. Group II (GII) received the same treatment as GI, however, received midazolam at $0.2 \mathrm{mg} \mathrm{kg}^{-1}$ in combination with dexmedetomidine, and another dose of $0.2 \mathrm{mg} \mathrm{kg} \mathrm{h}^{-1}$, in combination with dexmedetomidine and ketamine along with one hour maintenance. Group III (GIII) received the same treatment as GII, but diazepam was used instead of midazolam as induction dose at $0.5 \mathrm{mg} \mathrm{kg-1}$, and $0.5 \mathrm{mg} \mathrm{kg} \mathrm{h}^{-1}$ in the maintenance dose. Benzodiazepines effects were evaluated forward the cataleptic effects (contraction) of ketamine over dexmedetomidine. The respiratory and oxycapnomethetic parameters, bispectral index and recuperation period were also evaluated. After collection of the data, the statistic evaluation was done by Morrison's multivariate statistical methods. It was possible to conclude that the benzodiazepines combination showed synergistic interaction in sedation and muscle relaxant, with cardiorespiratory stability after

${ }^{1}$ Curso de Mestrado, Faculdade de Medicina Veterinária e Zootecnia (FMVZ), Universidade Estadual Paulista “Julio de Mesquita Filho" (UNESP), Botucatu, SP, Brasil. Rua Rubens Trefiglio, n.426, 13088-037, Campinas, SP, Brasil. E - mail: eduardohvet@gmail.com.

Autor para correpondência.

${ }^{2}$ Departamento de Cirurgia e Anestesiologia, FMVZ, UNESP “Julio de Mesquita Filho", Botucatu, SP, Brasil E - mail: btflama@uol.com.br

${ }^{3}$ Curso de Doutorado, Faculdade de Medicina de Botucatu, UNESP “Julio de Mesquita Filho", Botucatu, SP Brasil. 
pretreated with atropine, being a great value as in containment pharmacology.

Key words: dexmedetomidine, ketamine, benzodiazepines, continuous infusion, dog.

\section{INTRODUÇÃO}

A dexmedetomidina é um isômero farmacologicamente ativo da medetomidina, altamente lipofílica que demonstrou alta seletividade pelos receptores $\alpha_{2}$ - adrenérgicos, mais do que a clonidina (ANGELINI et al., 2000), e quando estimulados, inibem a liberação de noradrenalina e a atividade simpática, diminuem a pressão arterial e a freqüência cardíaca e promovem sedação, ansiólise e analgesia. (MANTZ, 2000).

Após a infusão,a dexmedetomidina apresenta uma fase rápida de distribuição com meia vida de aproximadamente 6 minutos, com ligação às proteinas plasmáticas de $93,7 \%$ e sem o comprometimento renal. Já o comprometimento hepático pode apresentar alterações nesta taxa, resultando em valores menores de depuração. O dexmedetomidina sofre uma ampla biotransformação no fígado e é excretada pela urina $(95 \%)$ e nas fezes (4\%). Seu período de latência é de aproximadamente 10 minutos após infusão, e o período hábil anestésico dura em torno de 4 horas (ANGELINI et al., 2000).

Evita-se a administração de injeções "em bolus", para minimizar os efeitos colaterais indesejáveis, como bradicardia e hipotensão, além de algumas arritmias nodais com aumento do tônus vagal (BLOOR et al., 1992). Esses efeitos adversos são reversíveis com a administração de atropina (SCHEININ et al., 1987). HATSCHBACH et al. (2003) demonstraram que a dexmedetomidina quando associada à quetamina oferece uma analgesia maior, entretanto não é suficiente para neutralizar por completo os efeitos cataleptóides da quetamina.

A atropina pode ser injetada por via intramuscular ou intravenosa quando se requer uma ação mais rápida e de efeito fugaz, sendo requerida a via subcutânea para efeitos mais prolongados, mas com período de latência de 15 minutos. A dose normalmente empregada é de $0,044 \mathrm{mg} \mathrm{kg}^{-1}$, não sendo aconselhável no cão, exceder a dose total de 1 a $1,5 \mathrm{mg}$, pois não possui antagonista (MASSONE,2003).

A quetamina é um fármaco dissociativo capaz de dissociar o córtex cerebral, causando analgesia e "desligamento", sem perda, porém, dos reflexos protetores. HASKINS et al., em 1985, observaram um período curto de anestesia, tônus muscular exacerbado, salivação e atividade muscular espontânea aumentada com o uso de quetamina isolada em cães. THURMON (1996) sugere que a quetamina não deve ser aplicada como agente anestésico isolado, pois, apesar de possuir uma baixa analgesia visceral, possui uma boa analgesia somática e causa uma ação cataleptóide sugerindo a associação com outros produtos que antagonizem seus efeitos estimulantes simpáticos. Os autores ainda notaram uma boa estabilidade cardíaca mais estável na associação acepromazina + buprenorfina e bradicardia acentuada nas demais associações.

Os benzodiazepínicos exibem efeitos ansiolíticos, tranquilizantes, hipnóticos, miorrelaxantes e provocam amnésia e alterações psicomotoras (RANDALL et al., 1974), agindo fundamentalmente sobre o sistema límbico reduzindo a atividade funcional do hipotálamo e córtex, com ação do ácido gamaaminobutírico (GABA) como neurotransmissor inibitor do sistema nervoso central (SPINOSA et al., 2002). O diazepam e o midazolam são os dois agentes mais empregados, sendo o midazolam um fármaco de meia vida (1,7 hora) mais curta que a diazepam com maior potência hipnótica (FANTONI \& CORTOPASSI, 2002).

Para se avaliar a profundidade do plano anestésico, tem se utilizado no homem, um monitor de ondas eletroencefalográficas que determina um índice conhecido como índice bispectral (BIS). Este índice é dimensionado em escala variando de 0 a 100 (unidades), na qual se observa uma diminuição à medida que o grau de sedação aumenta (ANTOGNINI et al., 2000).

Objetivou-se avaliar a parametria numa contenção farmacológica, observando-se os efeitos das associações atropina, dexmedetomidina, quetamina, midazolam ou diazepam sobre o animal; estudar o efeito de cada benzodiazepínico frente à associação utilizada, avaliando os atributos cardiorrespiratórios, índice bispectral e eletromiografia, e ainda, a resposta aos estímulos nociceptivos térmico e pressórico e a qualidade de recuperação.

\section{MATERIAL E MÉTODOS}

Foram empregados 30 cães hígidos (13 machos e 17 fêmeas), com pesos de 10 a $15 \mathrm{~kg}$ e idades de 2 a 4 anos, sem raça definida, evitando-se fêmeas prenhes ou em cio, previamente vermifugados e submetidos a exames clínicos e laboratoriais (bioquímico). Os animais provenientes do Canil Central da Faculdade de Medicina Veterinária e Zootecnia da Universidade estadual Paulista "Júlio de Mesquita Filho", distribuídos aleatoriamente em três grupos uniformes (GI, GII e GIII) com dez animais cada $(n=10)$, foram submetidos ao jejum alimentar de 12 horas e hídrico de 6 horas. 
O grupo I (GI) recebeu um pré-tratamento com atropina ${ }^{\mathrm{a}}$, na dose de $0,044 \mathrm{mg} \mathrm{kg}^{-1}$ pela via subcutânea na região costal direita, e após 15 minutos recebeu a dexmedetomidina ${ }^{\mathrm{b}}$ na dose de $3 \mu \mathrm{g} \mathrm{kg}^{-1}$ pela via intravenosa, em dose única, aplicada durante dois minutos. Logo após a indução, iniciou-se a manutenção com dexmedetomidina na dose de $3 \mu \mathrm{g} \mathrm{kg} \mathrm{h}^{-1}$, associada à quetamina ${ }^{\mathrm{c}}$ na dose de $2 \mathrm{mg} \mathrm{kg} \mathrm{h}^{-1}$, na mesma seringa. Completou-se com solução fisiológica até atingir-se $20 \mathrm{ml}$ de solução total, para que durante os próximos sessenta minutos, o animal recebesse esta associação por infusão continua através de bomba de seringa eletrônica ${ }^{\mathrm{d}}$, perfazendo uma hora de anestesia. O grupo II (GII) recebeu o mesmo tratamento de GI, recebendo, porém, o midazolam ${ }^{\mathrm{e}}$ na dose de $0,2 \mathrm{mg} \mathrm{kg}^{-1}$ associado à dexmedetomidina na dose de indução, aplicada durante dois minutos e outra dose, de $0,2 \mathrm{mg} \mathrm{kg} \mathrm{h}^{-1}$ associado à dose de manutenção da dexmedetomidina e quetamina. O grupo III (GIII) recebeu o mesmo tratamento de GII só que o midazolam foi substituído pelo diazepam ${ }^{\mathrm{f}}$, na dose de $0,5 \mathrm{mg} \mathrm{kg} .^{-1}$ na dose de indução e $0,5 \mathrm{mg} \mathrm{kg} \mathrm{h}^{-1}$ na dose de manutenção.

Os momentos mensurados foram: M0, animal sem aplicação do fármaco, M1, imediatamente antes da indução, M2, decorridos 10 minutos do início de M1, M3 decorridos 20 minutos e sucessivamente até M7. Ao final da manutenção anestésica, acompanhou-se a recuperação dos animais, registrando-se o período necessário para o aparecimento dos reflexos protetores, tônus postural, tentativa de deambulação e qualidade de recuperação anestésica (excitação, vocalização, tremores).

Em todos os momentos foram mensurados os seguintes parâmetros: temperatura retal em ${ }^{\circ} \mathrm{C}(\mathrm{TR})$, através de um termômetro clínico ${ }^{\mathrm{g}}$ com coluna de mercúrio; temperatura gengival $\mathrm{em}^{\circ} \mathrm{C}(\mathrm{TG})$, através de um termômetro a laser ${ }^{\mathrm{h}}$, leitura em três segundos; pressoalgimetria, mensurada em $\mathrm{kgf} \mathrm{cm}^{-2}$, pelo pressoalgimetro ${ }^{i}$, observando-se a resposta do animal frente à estimulação progressiva da pressão interdigital.

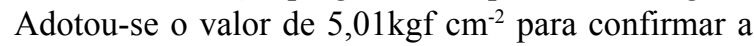
ausência de resposta ao estímulo pressórico, pois além destes limites caracterizava-se ausência de resposta, evitando-se assim, a lesão tissular; termoalgimetria, obtida em ${ }^{\circ} \mathrm{C}$ através do termoalgímetro ${ }^{\mathrm{j}}$, realizada através de um sensor aquecido a $52^{\circ} \mathrm{C}$ posicionado na membrana interdigital. Foram empregados períodos de estimulação até cinco segundos para se evitarem lesões tissulares (valores superiores eram considerados sem sensibilidade); freqüência cardíaca(FC), mensurada em batimentos por minuto (bpm) e a saturação de oxigênio $\left(\mathrm{SatO}_{2}\right)$, em \%, foram obtidos através do sensor de oximetria e pulso cardíaco do oxicapnógrafo ${ }^{\mathrm{k}}$; pressão arterial (PA), foi mensurada a pressão arterial sistólica (PAS), pressão arterial média (PAM) e a pressão arterial diastólica (PAD), em milímetros de mercúrio (mmHg), pelo método não invasivo, obtidas através da colocação de um manguito com largura proporcional (40\% do perímetro do membro) ao membro anterior, região proximal do úmero e conectado ao monitor'; freqüência respiratória $(f)$, através do sensor de capnometria próximo da narina do animal, utilizando-se um adaptador em forma de cone, mensurada em movimentos por minuto (mpm) pelo aparelho de oxicapnografia; concentração de dióxido de carbono no final da expiração $\left(\mathrm{ETCO}_{2}\right)$ e concentração de oxigênio no final da expiração $\left(\mathrm{ETO}_{2}\right) \mathrm{em} \mathrm{mmHg}$; fração inspirada de dióxido de carbono $\left(\mathrm{FiCO}_{2}\right)$ e fração inspirada de oxigênio $\left(\mathrm{FiO}_{2}\right)$, em \%; volume corrente $\left(\mathrm{V}_{\mathrm{T}}\right)$ e volume minuto $\left(\mathrm{V}_{\mathrm{M}}\right)$, em L/min, através da máscara adaptada às vias aéreas do animal, monitorada pelo oxicapnógrafo. A análise bispectral (BIS), leitura direta do monitor do aparelho de eletroencefalograma microprocessado ${ }^{\mathrm{m}}$ foi obtida através da colocação de dois eletrodos frontais e um temporal, sendo o resultado numérico avaliado através da escala bispectral: 0 - EEG isoelétrico (supressão) até 40 - hipnose profunda, 40 a 60 anestesia geral (hipnose moderada), 60 a 70 - sedação profunda (hipnose leve), 70 a 90 - sedação leve a moderada 90 a 100 - acordado. E eletromiografia (EMG), leitura direta em porcentagem no monitor do aparelho de índice bispectral microprocessado (BIS), avaliada através da escala de 0 a 100 , onde $0=$ miorrelaxamento completo, e 100 = animal com tônus muscular normal.

A avaliação estatística foi efetuada, após o término da colheita dos dados, por meio de Análise de Perfil (MORRISON, 1967 e CURI, 1980) considerando valores de $\mathrm{P}<0,05$ como níveis de significância, para interpretação dos possíveis efeitos que levariam as alterações nas médias de cada variável estudada nos diversos momentos, incluindo os testes das hipóteses de nulidade entre os momentos dentro de cada grupo, entre os momentos e entre grupos.

\section{RESULTADOS E DISCUSSÃO}

Avaliou-se a associação de dexmedetomidina, quetamina, midazolam ou diazepam, após pré-tratamento com atropina. Segundo HATSCHBACH et al. (2003), na indução, a dexmedetomidina associada à quetamina, não foi ideal como contenção farmacológica pois o efeito cataleptóide da quetamina se sobressaia sobre o efeito dos agonistas $\alpha_{2}$.

Diante de testes pilotos, em que se havia mudado a metodologia, subtraindo a quetamina na 
indução e passando-se a usar a dexmedetomidina isoladamente, notaram-se a ausência do efeito cataleptóide associando à quetamina apenas na manutenção, observando-se somente pequenos tremores musculares. Esta nova conduta comprovou que, ao se tolher a quetamina na indução e introduzindose as benzodiazepinas (midazolam e diazepam), cessaram as convulsões comprovando, assim, o miorrelaxamento de ação central (MASSONE, 2003)

A temperatura retal (TR) oscilou entre 37 a $39^{\circ} \mathrm{C}$, valores estes que estão próximos aos normais, não representando alterações fisiológicas. Vale ressaltar que estes animais não foram submetidos a qualquer tipo de sistema de aquecimento. Apenas a mesa de experimentação foi forrada com pano cirúrgico para maior conforto do animal.

A diminuição da temperatura após $\mathrm{M} 1$, em todos os grupos (Tabelas 1, 2 e 3), é o resultado de vários fatores como a exposição ao ambiente da sala de experimentação, depressão dos mecanismos termorreguladores provocados pela anestesia (SESSLER, 2000), como a vasodilatação periférica, redução do metabolismo basal, concomitantemente com a perda de calor pelas vias respiratórias (THURMON et al., 1996; SPINOSA et al., 2002). A temperatura gengival assumiu um comportamento semelhante, apesar de os valores ficarem abaixo dos valores da temperatura retal, também não foi de relevância clínica ou fisiológica, fato este motivado pela exposição gengival ao meio ambiente.

Após M1, a pressoalgimetria mostrou-se estável no decorrer dos momentos, mas, apesar dos grupos serem semelhantes, notou-se que, nas associações com benzodiazepínicos (Tabelas 1 e 2), a perda da sensibilidade foi maior. Esse fato é explicado pela ação dos benzodiazepínicos que reduzem a atividade funcional do hipotálamo e córtex, com ação no GABA, inibindo o sistema nervoso central e promovendo ação tranqüilizante, hipnótica, ansiolítica e miorrelaxante (SPINOSA et al., 2002). A termoalgimetria teve um comportamento análogo à pressoalgimetria.

A freqüência cardíaca foi semelhante em todos os grupos. A princípio ocorreu uma elevação observada nos momentos de M0 a M1, fato explicado pela administração da atropina 15 minutos após $\mathrm{M} 0 \mathrm{ou}$ imediatamente antes de M1. Logo após M1, houve uma redução gradativa da freqüência cardíaca até o último momento. O que se observou, em todos os grupos estudados (Tabelas 1, 2 e 3), é que a administração subcutânea da atropina na dose de $0,044 \mathrm{mg} \mathrm{kg}^{-1}$, como medicação pré-anestésica, previne o aparecimento da bradicardia, apesar da diminuição gradativa da freqüência, pois estes efeitos adversos como bradicardia, parada sinusal ou bloqueio atrioventricular são, segundo SCHEININ et al. (1987), reversíveis com a administração de atropina.

Foi observado um efeito bifásico da pressão arterial em todos os grupos, e logo após o início da administração dos fármacos houve aumento das pressões arteriais sistólica, média e diastólica. Nos momentos seguintes, foi notada redução gradativa das pressões arteriais até o momento final. Este efeito pode ser comprovado pela ação dos agonistas $\alpha_{2}$ impuros como a dexmedetomidina cuja atividade engloba tanto a ação a1 como $\alpha_{2}$, justificando a elevação inicial da pressão arterial seguida da sua queda (ação $\alpha 2$ )

$\mathrm{O}$ que se observou foi que nos grupos em que se associaram os benzodiazepínicos (GII e GIII) (Tabelas 2 e 3), a pressão arterial não se elevou tanto quanto ao grupo administrado apenas com a dexmedetomidina (GI) (Tabela 1). Este fato pode ser explicado, pois segundo JONES et al. (1979), os benzodiazepínicos ocasionam ligeira queda da pressão arterial, decorrente da redução da resistência vascular periférica.

Em geral, não houve alterações significativas dos atributos respiratórios no decorrer do experimento. Segundo MANTZ (2000), evidências sugerem que a dexmedetomidina não deprime significantemente a freqüência respiratória e a ventilação espontânea.

Foi observado em relação ao grupo I(Tabela 1), uma diminuição do índice bispectral apenas, logo após a aplicação da dexmedetomidina e início da infusão deste fármaco associada à quetamina, mesmo assim, atingindo valores de no máximo 91, e posteriormente obtendo valores maiores no decorrer dos momentos. Isto comprova que esta associação não promove um grau de sedação adequado. Nestes valores, os animais foram facilmente despertados quando submetidos a algum estímulo verbal ou nociceptivo. Considerando que o referido índice avalia a atividade cortical, e tendo em vista o mecanismo dissociativo da quetamina, esta, teoricamente, não influencia na redução do índice bispectral (HALL et al., 2001). KUREHARA et al. (1999) verificaram, após a administração de quetamina durante hipnose induzida por propofol, elevação do índice bispectral, sendo considerado por WU et al. (2001), um parâmetro não aplicável a pacientes submetidos à anestesia apenas com quetamina.

Ambos, agonistas $\alpha_{2}$ e benzodiazepínicos, exercem efeitos sedativos e ansiolíticos quando administrados como pré-tratamento. Com a associação 
Tabela 1 - Variação dos valores médios expressos em média $(\bar{X})$ e desvio padrão (S) (entre parênteses) em cães (n=10) pré-tratados com atropina e tratados com dexmedetomidina e quetamina (Grupo I).

\begin{tabular}{|c|c|c|c|c|c|c|c|c|}
\hline \multirow{3}{*}{ Variáveis } & \multicolumn{8}{|c|}{ Momentos } \\
\hline & s/fármaco & c/MPA. & $10 \mathrm{~min}$. & $20 \mathrm{~min}$. & $30 \mathrm{~min}$. & $40 \mathrm{~min}$. & $50 \mathrm{~min}$. & $60 \mathrm{~min}$. \\
\hline & M0 & M1 & M2 & M3 & M4 & M5 & M6 & M7 \\
\hline $\mathrm{T}^{\circ} \mathrm{C}$ retal $*$ & $39.0( \pm 0) \mathrm{a}$ & $39,1( \pm 0) \mathrm{a}$ & $38,8( \pm 0) \mathrm{a}$ & $38,7( \pm 0) b$ & $38,5( \pm 0) \mathrm{c}$ & $38,3( \pm 0) \mathrm{d}$ & $38,2( \pm 0) \mathrm{e}$ & $38,0( \pm 0) \mathrm{f}$ \\
\hline $\mathrm{T}^{\circ} \mathrm{C}$ gengival * & $38,0( \pm 1) \mathrm{a}$ & $37,9( \pm 1) \mathrm{a}$ & $38,1( \pm 0) \mathrm{a}$ & $38,0( \pm 1) \mathrm{a}$ & $38,0( \pm 0) \mathrm{a}$ & $37,9( \pm 0) b$ & $37,4( \pm 0) \mathrm{c}$ & $37,5( \pm 1) d$ \\
\hline Presso alg. * & $1,2( \pm 0) \mathrm{a}$ & $2,2( \pm 1) \mathrm{a}$ & $2,2( \pm 1) \mathrm{a}$ & $2,0( \pm 1) \mathrm{a}$ & $2,0( \pm 1) \mathrm{a}$ & $2,0( \pm 1) \mathrm{a}$ & $2,7( \pm 1) \mathrm{a}$ & $2,3( \pm 1) \mathrm{a}$ \\
\hline Termo alg. & $1,4( \pm 0) \mathrm{a}$ & $3,0( \pm 2) b$ & $3,1( \pm 1) b$ & $2,9( \pm 1) b$ & $2,8( \pm 1) b$ & $2,2( \pm 1) b$ & $2,0( \pm 1) b$ & $2,0( \pm 1) b$ \\
\hline $\mathrm{FC} *$ & $114( \pm 10) \mathrm{a}$ & $148( \pm 22) b$ & $143( \pm 20) b$ & $129( \pm 21) b$ & $119( \pm 16) b$ & $109( \pm 14) \mathrm{c}$ & $99( \pm 12) d$ & $92( \pm 8) \mathrm{e}$ \\
\hline PAS * & $132( \pm 11) \mathrm{a}$ & $205( \pm 11) b$ & $192( \pm 10) \mathrm{c}$ & $176( \pm 10) \mathrm{c}$ & $168( \pm 9) d$ & $157( \pm 11) \mathrm{e}$ & $147( \pm 15) \mathrm{f}$ & $149( \pm 16) g$ \\
\hline PAM & $103( \pm 8) \mathrm{a}$ & $169( \pm 6) b$ & $153( \pm 13) \mathrm{c}$ & $147( \pm 8) \mathrm{c}$ & $141( \pm 7) \mathrm{c}$ & $134( \pm 5) \mathrm{c}$ & $124( \pm 7) \mathrm{d}$ & $122( \pm 9) \mathrm{e}$ \\
\hline $\mathrm{PAD} *$ & $68( \pm 10) \mathrm{a}$ & $131( \pm 10) b$ & $126( \pm 12) \mathrm{c}$ & $116( \pm 14) \mathrm{c}$ & $112( \pm 17) \mathrm{c}$ & $110( \pm 13) \mathrm{c}$ & $101( \pm 18) \mathrm{c}$ & $92( \pm 13) d$ \\
\hline $\mathrm{SatO}_{2} *$ & $95( \pm 3) \mathrm{a}$ & $95( \pm 3)$ a & $95( \pm 1) \mathrm{a}$ & $95( \pm 2) \mathrm{a}$ & $94( \pm 2) \mathrm{a}$ & $93( \pm 2)$ a & $94( \pm 2) \mathrm{a}$ & $94( \pm 2) \mathrm{a}$ \\
\hline$f^{*}$ & $18( \pm 4) \mathrm{a}$ & $22( \pm 8) \mathrm{a}$ & $21( \pm 8) \mathrm{a}$ & $19( \pm 5) \mathrm{a}$ & $15( \pm 3) \mathrm{a}$ & $16( \pm 3) \mathrm{a}$ & $16( \pm 5) \mathrm{a}$ & $16( \pm 5) \mathrm{a}$ \\
\hline $\mathrm{ETCO}_{2} *$ & $37( \pm 4) \mathrm{a}$ & $36( \pm 4) \mathrm{a}$ & $35( \pm 4) \mathrm{a}$ & $35( \pm 4) \mathrm{a}$ & $38( \pm 5) \mathrm{a}$ & $35( \pm 5)$ a & $33( \pm 6)$ a & $36( \pm 4)$ a \\
\hline $\mathrm{ETO}_{2} *$ & $16( \pm 1) \mathrm{a}$ & $15( \pm 1) \mathrm{a}$ & $16( \pm 1) \mathrm{a}$ & $15( \pm 1) \mathrm{a}$ & $15( \pm 1) \mathrm{a}$ & $16( \pm 1) \mathrm{a}$ & $16( \pm 1) \mathrm{a}$ & $16( \pm 1) \mathrm{a}$ \\
\hline $\mathrm{FiCO}_{2} *$ & $1,6( \pm 0) \mathrm{a}$ & $2,1( \pm 1) \mathrm{a}$ & $1,7( \pm 1) \mathrm{a}$ & $1,9( \pm 1) \mathrm{a}$ & $1,7( \pm 0) \mathrm{a}$ & $1,8( \pm 1) \mathrm{a}$ & $1,9( \pm 1) \mathrm{a}$ & $1,7( \pm 0) \mathrm{a}$ \\
\hline $\mathrm{FiO}_{2} *$ & $21( \pm 0) \mathrm{a}$ & $20( \pm 0,3) \mathrm{a}$ & $21( \pm 0) \mathrm{a}$ & $21( \pm 0) \mathrm{a}$ & $21( \pm 0) \mathrm{a}$ & $21( \pm 0) \mathrm{a}$ & $21( \pm 0) \mathrm{a}$ & $21( \pm 0) \mathrm{a}$ \\
\hline $\mathrm{V}_{\mathrm{T}} *$ & $227( \pm 55) \mathrm{a}$ & $197( \pm 44) \mathrm{a}$ & $179( \pm 43) \mathrm{a}$ & $189( \pm 20) \mathrm{a}$ & $192( \pm 38) \mathrm{a}$ & $182( \pm 27) \mathrm{a}$ & $212( \pm 27) \mathrm{a}$ & $196( \pm 27) \mathrm{a}$ \\
\hline $\mathrm{V}_{\mathrm{M}} *$ & $4,1( \pm 1) \mathrm{a}$ & $4,3( \pm 1) \mathrm{a}$ & $3,6( \pm 1) \mathrm{a}$ & $3,5( \pm 1) \mathrm{a}$ & $2,9( \pm 1) \mathrm{a}$ & $2,9( \pm 1)$ a & $3,4( \pm 1)$ a & $3,1( \pm 1) \mathrm{a}$ \\
\hline BIS & $98( \pm 0) \mathrm{a}$ & $91( \pm 0) b$ & $92( \pm 4) b$ & $97( \pm 1) \mathrm{c}$ & $97( \pm 2) c$ & $97( \pm 2) c$ & $98( \pm 1) \mathrm{c}$ & $98( \pm 0) \mathrm{c}$ \\
\hline EMG & $98( \pm 2) \mathrm{a}$ & $49( \pm 7) b$ & $54( \pm 10) \mathrm{c}$ & $57( \pm 12) c$ & $69( \pm 23) \mathrm{c}$ & $75( \pm 23) c$ & $81( \pm 21) \mathrm{c}$ & $80( \pm 20) \mathrm{c}$ \\
\hline
\end{tabular}

As letras representam a igualdade entre os momentos. Para cada variável, médias seguidas pela mesma letra, não diferem significativamente pela análise de perfil $(\mathrm{P}>0,05)$.

Os símbolos (na coluna das variáveis) representam a igualdade entre grupos, relacionada com as tabelas 2 e 3 . Mesmas variáveis com símbolos, não diferem significativamente pela análise de perfil $(\mathrm{P}>0,05)$.

Temperatura retal em ${ }^{\circ} \mathrm{Celsius}\left(\mathrm{T}^{\circ} \mathrm{C}\right.$ retal); Temperatura gengival em ${ }^{\circ} \mathrm{C}\left(\mathrm{T}^{\circ} \mathrm{C}\right.$ gengival); Presso algimetria em $\mathrm{kgf} / \mathrm{cm}^{2}(\mathrm{Presso}$ alg); $\mathrm{Termo}$ algimetria em segundos (Termo alg.); Freqüência cardíaca em bpm (FC); Pressão arterial sistólica em mmHg (PAS); Pressão arterial média em mmHg (PAM); Pressão arterial diastólica em mmHg (PAD); Saturação de oxigênio em \% $\left(\right.$ SatO $\left._{2}\right)$; Freqüência respiratória mpm $(f)$; Concentração de dióxido de carbono no final da expiração em $\mathrm{mmHg}\left(\mathrm{ETCO}_{2}\right)$; Concentração de oxigênio no final da expiração em mmHg $\left(\mathrm{ETO}_{2}\right)$; Fração inspirada de dióxido de carbono em \% $\left(\mathrm{FiCO}_{2}\right)$; Fração inspirada de oxigênio em \% $\left.(\mathrm{FiO})_{2}\right)$, Volume corrente em ml $\left(\mathrm{V}_{\mathrm{T}}\right)$; Volume minuto em L/min ( $\mathrm{V}_{\mathrm{M}}$ ); Índice bispectral em unidade (BIS); Eletromiografia em \% (EMG).

destes dois fármacos, dexmedetomidina a $0,5 \mu \mathrm{g} \mathrm{kg}{ }^{-1} \mathrm{e}$ midazolam a $0,5 \mathrm{mg} \mathrm{kg}^{-1}$, há um sinergismo (SALONEN et al., 1992). HORVATH et al. (1992) verificaram em uma associação com dexmedetomidina e diazepam que, em ambos os fármacos, menos de um quarto de suas doses isoladas, eram necessários para produzir o mesmo efeito, confirmando assim, um sinergismo capaz de produzir uma hipnose maior pela ação da dexmedetomidina e o diazepam.
Nota-se que existe um sinergismo maior na sedação, quando os benzodiazepínicos são associados à dexmedetomidina (Tabelas 2 e 3). Este sinergismo foi maior com o uso do midazolam em GII (Tabela 2), resultando em valores menores do BIS (70). Isto se deve ao fato de que o midazolam possui uma ação hipnótica mais potente quando comparado ao diazepam (FANTONI \& CORTOPASSI, 2002). 
Tabela 2 - Variação dos valores médios expressos em média $(\bar{X})$ e desvio padrão (S) (entre parênteses) em cães (n=10) pré-tratados com atropina e tratados com dexmedetomidina, quetamina e midazolam (Grupo II).

\begin{tabular}{|c|c|c|c|c|c|c|c|c|}
\hline \multirow{3}{*}{ Variáveis } & \multicolumn{8}{|c|}{ Momentos } \\
\hline & s/fármaco & c/MPA. & $10 \mathrm{~min}$. & $20 \mathrm{~min}$. & $30 \mathrm{~min}$. & $40 \mathrm{~min}$. & $50 \mathrm{~min}$. & $60 \mathrm{~min}$. \\
\hline & M0 & M1 & M2 & M3 & M4 & M5 & M6 & M7 \\
\hline $\mathrm{T}^{\circ} \mathrm{C}$ retal $*$ & $38,8( \pm 0) \mathrm{a}$ & $39,0( \pm 0) \mathrm{a}$ & $38,8( \pm 0) \mathrm{a}$ & $38,6( \pm 0) \mathrm{a}$ & $38,4( \pm 0) \mathrm{a}$ & $38,3( \pm 1) b$ & $38,1( \pm 1) \mathrm{c}$ & $38,1( \pm 1) \mathrm{d}$ \\
\hline $\begin{array}{l}\mathrm{T}^{\circ} \mathrm{C} \text { gengival } \\
* \text {. }\end{array}$ & $37,7( \pm 0) \mathrm{a}$ & $38,0( \pm 1) \mathrm{a}$ & $38,1( \pm 1) \mathrm{a}$ & $37,9( \pm 1) \mathrm{a}$ & $37,8( \pm 1) a$ & $37,7( \pm 1) \mathrm{a}$ & $37,7( \pm 1) \mathrm{a}$ & $37,6( \pm 1) \mathrm{a}$ \\
\hline Presso alg. * & $1,0( \pm 0) \mathrm{a}$ & $3,1( \pm 1) b$ & $2,9( \pm 1) b$ & $3,5( \pm 1) \mathrm{c}$ & $2,9( \pm 1) c$ & $3,3( \pm 1) \mathrm{c}$ & $3,2( \pm 1) \mathrm{c}$ & $3,0( \pm 1) \mathrm{c}$ \\
\hline Termo alg. * & $1,9( \pm 0) \mathrm{a}$ & $4,0( \pm 1) b$ & $4,3( \pm 1) \mathrm{c}$ & $4,6( \pm 1) \mathrm{d}$ & $4,8( \pm 0) \mathrm{e}$ & $4,6( \pm 1) \mathrm{e}$ & $3,9( \pm 1) \mathrm{e}$ & $4,0( \pm 1) \mathrm{e}$ \\
\hline $\mathrm{FC} *$ & $109( \pm 22) \mathrm{a}$ & $135( \pm 26) \mathrm{a}$ & $131( \pm 23) \mathrm{a}$ & $118( \pm 24) \mathrm{a}$ & $105( \pm 22) \mathrm{a}$ & $100( \pm 23)$ a & $93( \pm 21) \mathrm{a}$ & $92( \pm 22) \mathrm{a}$ \\
\hline PAS * & $116( \pm 13) \mathrm{a}$ & $170( \pm 36) b$ & $163( \pm 29) b$ & $144( \pm 26) b$ & $149( \pm 4,4) b$ & $146( \pm 13) b$ & $142( \pm 16) b$ & $143( \pm 15) b$ \\
\hline PAM * & $96( \pm 11) \mathrm{a}$ & $145( \pm 13) b$ & $129( \pm 14) \mathrm{c}$ & $114( \pm 13) \mathrm{c}$ & $118( \pm 12) \mathrm{c}$ & $117( \pm 13) \mathrm{c}$ & $115( \pm 23) \mathrm{c}$ & $116( \pm 17) \mathrm{c}$ \\
\hline PAD * & $76( \pm 15) \mathrm{a}$ & $122( \pm 21) b$ & $99( \pm 20) b$ & $90( \pm 18) b$ & $92( \pm 21) b$ & $94( \pm 14) b$ & $94( \pm 39) b$ & $92( \pm 20) b$ \\
\hline $\mathrm{SatO}_{2} *$ & $96( \pm 3) \mathrm{a}$ & $95( \pm 5) \mathrm{a}$ & $93( \pm 5) \mathrm{a}$ & $94( \pm 4) \mathrm{a}$ & $95( \pm 3) \mathrm{a}$ & $95( \pm 2) \mathrm{a}$ & $96( \pm 2) \mathrm{a}$ & $97( \pm 2) \mathrm{a}$ \\
\hline$f^{*}$ & $23( \pm 6) \mathrm{a}$ & $28( \pm 5) \mathrm{a}$ & $27( \pm 8) \mathrm{a}$ & $24( \pm 9) \mathrm{a}$ & $24( \pm 10)$ a & $22( \pm 10) \mathrm{a}$ & $21( \pm 9) \mathrm{a}$ & $21( \pm 9) \mathrm{a}$ \\
\hline $\mathrm{ETCO}_{2} *$ & $34( \pm 6) \mathrm{a}$ & $29( \pm 6) \mathrm{a}$ & $33( \pm 8) \mathrm{a}$ & $34( \pm 8) \mathrm{a}$ & $34( \pm 8) \mathrm{a}$ & $35( \pm 8)$ a & $33( \pm 9) \mathrm{a}$ & $32( \pm 9)$ a \\
\hline $\mathrm{ETO}_{2} *$ & $15( \pm 1) \mathrm{a}$ & $16( \pm 1) \mathrm{a}$ & $15( \pm 2) \mathrm{a}$ & $15( \pm 1) \mathrm{a}$ & $15( \pm 1) \mathrm{a}$ & $15( \pm 1) \mathrm{a}$ & $16( \pm 2) \mathrm{a}$ & $16( \pm 1) \mathrm{a}$ \\
\hline $\mathrm{FiCO}_{2} *$ & $1,4( \pm 0) \mathrm{a}$ & $2,2( \pm 1) \mathrm{a}$ & $1,6( \pm 0) \mathrm{a}$ & $1,4( \pm 0) \mathrm{a}$ & $1,8( \pm 1) \mathrm{a}$ & $1,7( \pm 1) \mathrm{a}$ & $1,7( \pm 0) \mathrm{a}$ & $1,3( \pm 0) \mathrm{a}$ \\
\hline $\mathrm{FiO}_{2} *$ & $21( \pm 0) \mathrm{a}$ & $20( \pm 0) \mathrm{a}$ & $20( \pm 0) \mathrm{a}$ & $21( \pm 0) \mathrm{a}$ & $21( \pm 0) \mathrm{a}$ & $21( \pm 0) \mathrm{a}$ & $20( \pm 0) \mathrm{a}$ & $21( \pm 0) \mathrm{a}$ \\
\hline $\mathrm{V}_{\mathrm{T}} *$ & $206( \pm 75) \mathrm{a}$ & $133( \pm 34) b$ & $149( \pm 52) b$ & $140( \pm 52) b$ & $152( \pm 65) b$ & $166( \pm 57) \mathrm{b}$ & $165( \pm 47) b$ & $157( \pm 47) b$ \\
\hline$V_{M} *$ & $5( \pm 3) \mathrm{a}$ & $3,7( \pm 1) \mathrm{a}$ & $3,8( \pm 1) \mathrm{a}$ & $3,2( \pm 1) \mathrm{a}$ & $3,4( \pm 2) \mathrm{a}$ & $3,3( \pm 1) \mathrm{a}$ & $3,3( \pm 1) \mathrm{a}$ & $3,1( \pm 1) \mathrm{a}$ \\
\hline BIS * & $98( \pm 0) \mathrm{a}$ & $84( \pm 9) b$ & $80( \pm 9) \mathrm{c}$ & $76( \pm 8) d$ & $72( \pm 8) \mathrm{e}$ & $71( \pm 8) \mathrm{f}$ & $75( \pm 8) \mathrm{f}$ & $75( \pm 11) \mathrm{f}$ \\
\hline EMG * & $96( \pm 4) \mathrm{a}$ & $26( \pm 15) b$ & $20( \pm 16) c$ & $20( \pm 12) d$ & $16( \pm 10) \mathrm{e}$ & $17( \pm 8) \mathrm{f}$ & $17( \pm 9) \mathrm{g}$ & $17( \pm 8) \mathrm{h}$ \\
\hline
\end{tabular}

As letras representam a igualdade entre os momentos. Para cada variável, médias seguidas pela mesma letra, não diferem significativamente pela análise de perfil $(\mathrm{P}>0,05)$.

Os símbolos (na coluna das variáveis) representam a igualdade entre grupos, relacionada com as tabelas 1 e 3 . Mesmas variáveis com símbolos, não diferem significativamente pela análise de perfil $(\mathrm{P}>0,05)$.

O índice bispectral, nos grupos II e III (Tabelas 2 e 3), variou entre 70 e 90, o que indica uma sedação de leve à moderada, que fazia com que os animais não fossem despertados, quando submetidos aos estímulos sonoros ou nociceptivos. Esse fato está de acordo com ANTOGININI et al. (2000), os quais afirmam que, com a diminuição do índice bispectral, aumenta o grau de hipnose.

Através da eletromiografia obtida pelo BIS, observou-se uma correlação em relação aos valores do índice bispectral. No grupo I (Tabela 1), observou-se uma porcentagem alta de atividade muscular, ou seja, não apresentando grau de miorrelaxamento adequado durante toda a manutenção. Já o relaxamento muscular de origem central produzido pelo agonista $\alpha 2$ (FANTONI \& CORTOPASSI, 2002) não foi suficiente quando associado à quetamina, provando que o tônus muscular excessivo, provocado pela quetamina se sobressai não somente sobre o efeito de sedação como também sobre o miorrelaxamento causado pela dexmedetomidina.

Foi observado um bom grau de relaxamento nos grupos II e III (Tabelas 2 e 3), obtendo-se mais uma vez, uma interação de sinergismo dos agonistas $\alpha_{2}$ com os benzodiazepínicos em relação aos efeitos 
Tabela 3 - Variação dos valores médios expressos em média $(\bar{X})$ e desvio padrão (S) (entre parênteses) em cães (n=10) pré-tratados com atropina e tratados com dexmedetomidina, quetamina e diazepam (Grupo III).

\begin{tabular}{|c|c|c|c|c|c|c|c|c|}
\hline \multirow{3}{*}{ Variáveis } & \multicolumn{8}{|c|}{ Momentos } \\
\hline & s/fármaco & $\mathrm{c} / \mathrm{MPA}$ & $10 \mathrm{~min}$. & $20 \mathrm{~min}$. & $30 \mathrm{~min}$. & $40 \mathrm{~min}$. & $50 \mathrm{~min}$. & $60 \mathrm{~min}$. \\
\hline & M0 & M1 & M2 & M3 & M4 & M5 & M6 & M7 \\
\hline $\mathrm{T}^{\circ} \mathrm{C}$ retal & $39.0( \pm 0) \mathrm{a}$ & $39,1( \pm 0) \mathrm{a}$ & $38,8( \pm 0) \mathrm{a}$ & $38,7( \pm 0) b$ & $38,5( \pm 0) \mathrm{c}$ & $38,3( \pm 0) \mathrm{d}$ & $38,2( \pm 0) \mathrm{e}$ & $38,0( \pm 0) \mathrm{f}$ \\
\hline $\mathrm{T}^{\circ} \mathrm{C}$ gengival. & $38,0( \pm 0) \mathrm{a}$ & $37,9( \pm 0) \mathrm{a}$ & $38,1( \pm 0) \mathrm{a}$ & $38,0( \pm 0) \mathrm{a}$ & $38,0( \pm 0) \mathrm{a}$ & $37,9( \pm 0) \mathrm{a}$ & $37,4( \pm 1) b$ & $37,5( \pm 1) \mathrm{c}$ \\
\hline Presso alg. * & $0,9( \pm 0) \mathrm{a}$ & $2,6( \pm 1) b$ & $2,8( \pm 1) \mathrm{c}$ & $2,5( \pm 1) \mathrm{c}$ & $2,8( \pm 1) \mathrm{c}$ & $2,6( \pm 1) \mathrm{c}$ & $2,1( \pm 0) \mathrm{c}$ & $2,2( \pm 1) \mathrm{c}$ \\
\hline Termo alg. * & $1,4( \pm 0) \mathrm{a}$ & $3,7( \pm 1) b$ & $4,3( \pm 1) \mathrm{c}$ & $3,8( \pm 1) \mathrm{c}$ & $3,6( \pm 1) c$ & $3,5( \pm 1) \mathrm{c}$ & $3,5( \pm 1) \mathrm{c}$ & $3,3( \pm 1) \mathrm{c}$ \\
\hline $\mathrm{FC} *$ & $120( \pm 12) \mathrm{a}$ & $137( \pm 33)$ a & $130( \pm 34) \mathrm{a}$ & $120( \pm 20) \mathrm{a}$ & $112( \pm 14) \mathrm{a}$ & $104( \pm 14) b$ & $96( \pm 14) \mathrm{c}$ & $94( \pm 13) d$ \\
\hline PAS & $115( \pm 22) \mathrm{a}$ & $175( \pm 23) b$ & $162( \pm 31) b$ & $147( \pm 35) b$ & $138( \pm 33) b$ & $119( \pm 23) \mathrm{c}$ & $128( \pm 25) \mathrm{c}$ & $116( \pm 26) d$ \\
\hline PAM * & $95( \pm 10)$ a & $143( \pm 14) b$ & $135( \pm 15) b$ & $122( \pm 22) b$ & $117( \pm 21) b$ & $101( \pm 12) \mathrm{c}$ & $108( \pm 19) \mathrm{c}$ & $100( \pm 16) d$ \\
\hline $\mathrm{PAD} *$ & $76( \pm 17) \mathrm{a}$ & $106( \pm 23) \mathrm{a}$ & $100( \pm 22) \mathrm{a}$ & $89( \pm 34)$ a & $90( \pm 25)$ a & $74( \pm 20)$ a & $79( \pm 29)$ a & $77( \pm 23)$ a \\
\hline $\mathrm{SatO}_{2} *$ & $97( \pm 1) \mathrm{a}$ & $94( \pm 2) \mathrm{a}$ & $94( \pm 2)$ a & $94( \pm 1)$ a & $94( \pm 2)$ a & $95( \pm 2)$ a & $95( \pm 2)$ a & $95( \pm 3)$ a \\
\hline$f^{*}$ & $20( \pm 4) \mathrm{a}$ & $29( \pm 8) \mathrm{a}$ & $27( \pm 8) \mathrm{a}$ & $25( \pm 8) \mathrm{a}$ & $22( \pm 7) \mathrm{a}$ & $22( \pm 8) \mathrm{a}$ & $21( \pm 8) \mathrm{a}$ & $20( \pm 6) \mathrm{a}$ \\
\hline $\mathrm{ETCO}_{2} *$ & $39( \pm 4) \mathrm{a}$ & $33( \pm 4) \mathrm{a}$ & $34( \pm 5)$ a & $35( \pm 5) \mathrm{a}$ & $36( \pm 5) \mathrm{a}$ & $35( \pm 6)$ a & $36( \pm 5)$ a & $35( \pm 5)$ a \\
\hline $\mathrm{ETO}_{2} *$ & $15( \pm 1) \mathrm{a}$ & $16( \pm 1) \mathrm{a}$ & $15( \pm 1) \mathrm{a}$ & $15( \pm 1) \mathrm{a}$ & $15( \pm 1) \mathrm{a}$ & $15( \pm 1) \mathrm{a}$ & $15( \pm 1) \mathrm{a}$ & $16( \pm 1) \mathrm{a}$ \\
\hline $\mathrm{FiCO}_{2} *$ & $1,8( \pm 2) \mathrm{a}$ & $1,7( \pm 1) \mathrm{a}$ & $1,7( \pm 0) \mathrm{a}$ & $1,3( \pm 0)$ a & $1,7( \pm 1) \mathrm{a}$ & $1,5( \pm 1) \mathrm{a}$ & $1,3( \pm 1) \mathrm{a}$ & $1,5( \pm 0) \mathrm{a}$ \\
\hline $\mathrm{FiO}_{2}$ * & $20( \pm 0) \mathrm{a}$ & $21( \pm 0) \mathrm{a}$ & $21( \pm 0) \mathrm{a}$ & $21( \pm 0) \mathrm{a}$ & $21( \pm 0) \mathrm{a}$ & $21( \pm 0) \mathrm{a}$ & $21( \pm 0) \mathrm{a}$ & $21( \pm 0) \mathrm{a}$ \\
\hline $\mathrm{V}_{\mathrm{T}}$ & $162( \pm 46) \mathrm{a}$ & $135( \pm 62) \mathrm{a}$ & $115( \pm 30)$ a & $124( \pm 40) \mathrm{a}$ & $120( \pm 34) \mathrm{a}$ & $138( \pm 41)$ a & $140( \pm 44) \mathrm{a}$ & $145( \pm 63) \mathrm{a}$ \\
\hline $\mathrm{V}_{\mathrm{M}} *$ & $3,3( \pm 1)$ a & $3,7( \pm 1) \mathrm{a}$ & $3( \pm 1)$ a & $3( \pm 1)$ a & $2,6( \pm 1) \mathrm{a}$ & $2,9( \pm 1) \mathrm{a}$ & $2,8( \pm 1) \mathrm{a}$ & $2,7( \pm 1) \mathrm{a}$ \\
\hline BIS * & $98( \pm 0) \mathrm{a}$ & $88( \pm 3)$ a & $83( \pm 8) b$ & $80( \pm 11) \mathrm{c}$ & $81( \pm 11) \mathrm{c}$ & $83( \pm 10) \mathrm{c}$ & $85( \pm 8) c$ & $88( \pm 8) \mathrm{c}$ \\
\hline EMG * & $96( \pm 4) \mathrm{a}$ & $39( \pm 16) b$ & $26( \pm 13) \mathrm{c}$ & $26( \pm 13) d$ & $26( \pm 14) d$ & $25( \pm 15) d$ & $27( \pm 18) d$ & $29( \pm 17) d$ \\
\hline
\end{tabular}

As letras representam a igualdade entre os momentos. Para cada variável, médias seguidas pela mesma letra não diferem significativamente pela análise de perfil $(\mathrm{P}>0,05)$.

Os símbolos (na coluna das variáveis) representam a igualdade entre grupos, relacionada com as tabelas 1e 2. Mesmas variáveis com símbolos não diferem significativamente pela análise de perfil $(\mathrm{P}>0,05)$.

miorrelaxantes de ação central, pois os mesmos se sobrepuseram aos efeitos cataleptóides causados pela quetamina (MASSONE, 2003).

Em relação ao período de recuperação, avaliada logo após o término da infusão (até que o animal estivesse em posição quadrupedal), notaramse valores muito próximos com diferenças de poucos minutos entre grupos. Os valores atingiram de 9 a 12 minutos, o que atribuiu uma qualificação de recuperação rápida, além da ausência de efeitos indesejáveis como vocalização, tremores, vômitos ou sialorréia também descrito por BEIER (2003).

\section{CONCLUSÕES}

Diante da metodologia empregada, concluise que a quetamina, quando aplicada após a dexmedetomidina, não causa atividade cataleptóide, porém, exibe pouca influência sobre a redução do índice bispectral. A associação do midazolam ou diazepam mostrou ser mais eficiente, com estabilidade cardiorrespiratória e um sinergismo na sedação e miorrelaxamento, mais evidente com o uso do midazolam, além de a recuperação mostrar-se mais tranqüila e sem qualquer efeito colateral. 
A administração dos benzodiazepínicos associados à atropina - dexmedetomidina - quetamina, é eficiente na contenção farmacológica de cães.

\section{FONTES DE AQUISIÇÃO}

a Hytropin - HypoFarma;

${ }^{\mathrm{b}}$ Prededex - Abbott; ' $\mathrm{C}$ Ketalar -Pfizer;

${ }^{\mathrm{d}}$ Samtronic do Brasil; ${ }^{\mathrm{e}}$ Dormonid - Roche;

${ }^{\mathrm{f}}$ Diazepamil - Hipolabor;

g Termômetro clínico digital B-D;

${ }^{\mathrm{h}}$ Raytec IOPE Instrumentos de Precisão Ltda (FAPESP n ${ }^{\circ} 2000$ 09838-7);

${ }^{i}$ Pressoalgimetro - IOPE (FAPESP n²000/09838);

'Termoalgimetro - IOPE (FAPESP n²000/09838-7);

${ }^{\mathrm{k}}$ Capnomac Ultima - Datex Engstron (FAPESP no00/09838-7);

${ }^{1}$ Monitor multiparamêtrico Dixtal (FAPESP n ${ }^{\circ} 2000 / 02583-3$ );

${ }^{m}$ BIS Monitor A-2000 - Aspect Medical System (FAPESP n ${ }^{\circ} 00$ / 09838-7).

Esta pesquisa foi aprovada pela Comissão de Ética na Experimentação Animal (CEEA) da Faculdade de Medicina Veterinária e Zootecnia UNESP - Campus de Botucatu, estando, portanto, de acordo com os princípios éticos adotados pelo Colégio Brasileiro de Experimentação Animal (COBEA).

\section{AGRADECIMENTOS}

À Fundação de Amparo à Pesquisa do Estado de São Paulo FAPESP pelo Auxílio à Pesquisa, o que permitiu o bom desenvolvimento deste trabalho.

\section{REFERÊNCIAS}

ANGELINI, G. et al. The effects of alpha-adrenoceptor agonists on circulation and respiration. Baill Clin Anesth, v.14, n.2, p.449-458, 2000.

ANTOGNINI, J.F. et al. Isofluorane anesthetic depth in goats monitored using bispectral index of the electroencephalogram. Vet Res Commun, v.24, n.6, p.361-370, 2000.

BEIER, S.L. Avaliação bispectral e nociceptiva da associação da dexmedetomidina em case, pré-tratados ou não pela atropina e tratados ou não pela buprenorfina. 2003. 154f. Dissertação (Mestrado em Anestesiologia) - Curso de Pós-graduação da Faculdade de Medicina Campus de Botucatu, Universidade Estadual Paulista "Júlio de Mesquita Filho".

BLOOR, B.C. et al. Hemodynamic and sedative effects of dexmedetomidina in dog. J Pharmacol Exp Ther, v.263, p.690-697, 1992 .

CURI, P.R. Análise de medidas repetidas em modelos biológicos. Rev Brás Estat, v.41, p.137-150, 1980

FANTONI, D.T.; CORTOPASSI, S.R.G. Anestesia em cães e gatos. Roca: São Paulo, 2002. 389p.
HALL, L.W. et al. Veterinary anesthesia. London: Baillière $\backslash$ Tindall, 2001. 561p.

HASKINS, S.C. et al. Ketamine in dogs. Am J Vet Res, v.46, p. $855-1860,1985$.

HATSCHBACH, E. et al. Avaliação para métrica do cloridrato de dexmedetomidina em cães pré - tratados ou não pela atropina e tratados ou não pela quetamina. ARS Veterinária, v.21, n.1, p.22-29, 2005

HORVATH, G. et al. An isobolografhic analysis of the effects of combinations of dexmedetomidina with fentanyl or diazepam in rats. Life-Sci, v.50, p.215-220, 1992.

JONES, D.J. et al. Cardiovascular responses to diazepam and midazolam maleate in the dos. Anesth, v.51, p.430-434, 1979.

KUREHARA, K. et al. The influence of ketamine o bispectral index, the spectral edge frequency 90 and the frequency bands power during propofol anesthesia. Masui, v.48, n.6, p.611616,1999

MANTZ, J. Alpha 2 adrenoceptor agonists: analgesia, sedation, anxiolysis, haemodynamics, respiratory function and weaning. Baill Clin Anesth, v.14, n.2, p.433-448, 2000

MASSONE, F. Anestesiologia veterinária: farmacologia e técnicas. 4.ed. Rio de Janeiro: Guanabara Koogan, 2003. $326 \mathrm{p}$.

MORRISON, D.F. Multivariate statistical methods. New York: McGrows Hill Books, 1967. 388p.

RANDALL, L.O. et al. Chemistry and pharmacology of the 1,4-benzodiazepines. Psychopharmacological Agents, New Jersey, v.3, p.01-34, 1974.

SALONEN, M. et al. Synergistic interaction between alpha 2 adrenergic agonists and benzodiazepines in rats. Anesthesiol, v.76, p.1004-1011, 1992.

SCHEININ, M. et al. Sedative and cardiovascular effects of medetomidine, a novel selective alpha ${ }_{2}$ adrenoceptor agonist, in healthy volunteers. Br J Clin Pharmacol. v.4, p.443451,1987

SESSLER, D.I. Temperature monitoring. In: MILLER, R.D. Anesthesia. 5.ed. Philadelphia: Churchill Livingstone, 2000 p.1367-1389.

SPINOSA, H.L.et al. Farmacologia aplicada à medicina veterinária. 3.ed. Rio de Janeiro: Guanabara Koogan, 2002. 752 p.

THURMON, J.C. et al. Preanesthetics and anesthetic adjuncts. In: THURMON, J.C. et al. Lumb \& Jones veterinary anesthesia. 3.ed. Baltimore: Willians \& Wilkins, 1996. Cap. 8, p.183-209.

WU, C.C. et al. EEG bispectral index changes with ketamine vesus thiamylal induction of anesthesia. Acta Anesthesiol, v.39, n.1, p.11-15, 2001. 